\title{
Socio-Economic Factors and Women Participation in Mining: a Case of Rwanda Mining
}

\author{
Patricie Mwambarangwe $^{1^{*}}$ Dr. Jaya Shukla ${ }^{2}$ \\ 1. Mount Kenya University, Kigali, Rwanda \\ 2. Mount Kenya University, Kigali Rwanda \\ * E-mail of the corresponding author:m.patricie370@gmail.com
}

\begin{abstract}
This report presents a study conducted in Rwanda Mining Association to assess Socio-Economic Factors and Women Participation in Rwandan Mining Projects. Specific objectives for the study were: To investigate the effect of socio-cultural and economic factors on women participation in mining projects; Determine the influence of company management procedures on women participation in mining project; and to examine the impact of mine sites working and living conditions on women participation in mining projects. Using purposive and simple random sampling technique, the target population of 835 workers (676 men and 159 women) was selected from 30 mining companies. The sample size of 270 respondents including 51 women and 219 men was also selected. Collected data was analysed through SPSS software using chi-square and regression models. It was found that socio-cultural beliefs and Socio-economic factors have negative relationship with women participation in mining. Company management procedures (recruitment, workers' promotion, and skills development and job allocation procedures) applied in human resources management were also found to have negative effect on women participation in mining. The nature of mining work places, working and living conditions in terms of occupational Safety and Health $(\mathrm{OSH})$ of workers do not facilitate women integration in mining. Female workers face particular challenges such as being fired once they get pregnant and lack of work contracts. If mining is to contribute for poverty reduction by eliminating inequality in accessing economic benefits in rural areas between men and women, all these factors should be addressed in a manner favourable to women. More sensitizations are recommended for community to promote good understanding on equal sharing of economic resources between men and women. Improved work environment is needed for both men and women. As in other sector of activity, women's rights associated with employment should be respected as well.
\end{abstract}

Key words: Culture, Gender equality; gender mainstreaming; Mining, Mining Project

DOI: $10.7176 / \mathrm{JESD} / 11-18-08$

Publication date:September $30^{\text {th }} 2020$

\section{Introduction}

Mining in Rwanda is considered as one of important economic activities expected to highly contribute for handling poverty in rural areas mainly caused by lack of equal consideration of men and women in accessing economic opportunities. In most cases women are deprived from economic benefits in rural areas while they constitute a majority of active population. (MINECOFIN,2013).

Within the extractive industry, uncovering reasons for low representation of women in mining workforce remains of big interest. Extractive industry's-specific workforces with related academic efforts have been found to be barriers that affect women engagement in the sector. (MIHRC,2016).

\section{Problem statement}

Mining projects have challenges, possibilities and risks to sustainable development for women. Even though some mining projects have policies on empowering women, equal employment opportunity and even with recognition of the quality of having men and women in mining projects workers, the effects of these policies present problems. The link between gender equality policies by mining operators and their actions are contradictory because mining projects continue to be male dominated businesses. (Pimpa, 2019). In the case of Rwanda, the mining sector has been set as one of important pillars for addressing the issue of poverty in rural areas caused by inequality in accessing economic resources between men and women where the later are vulnerable. (MINECOFIN,2013). Despite of strong government political will to strengthen gender equality at all levels, mining continues to be a male dominated sector. At the end of 2014, women were represented at $16 \%$ while in 2016 this number has reduced to $14 \%$. (RNRA,2016). 
Assessment of factors affecting women participation in mining projects starting from managerial, operational up to community levels would be an important input for setting up strong strategies to promote women integration in mining sector.

\section{Research objectives}

\subsection{General Objective}

The main objective of the research study was to examine factors which contribute to low participation of women in Rwandan mining projects.

\subsection{Specific objectives}

i. To investigate the effect of socio-cultural and economic factors on women participation in mining;

ii. To determine the influence of company management procedures on women participation in mining;

iii. To examine the impact of mine sites working and living conditions on women participation in mining.

\section{$4 \quad$ Research questions}

In order to realize research objectives and provide significant explanations to the research problem, the following questions were asked:

i. How do socio-cultural and economic factors affect participation of women in mining?

ii. How do company management procedures influence women participation in mining?

iii. How do working and living conditions of mine work place affect women participation in mining?

\section{Conceptual framework}

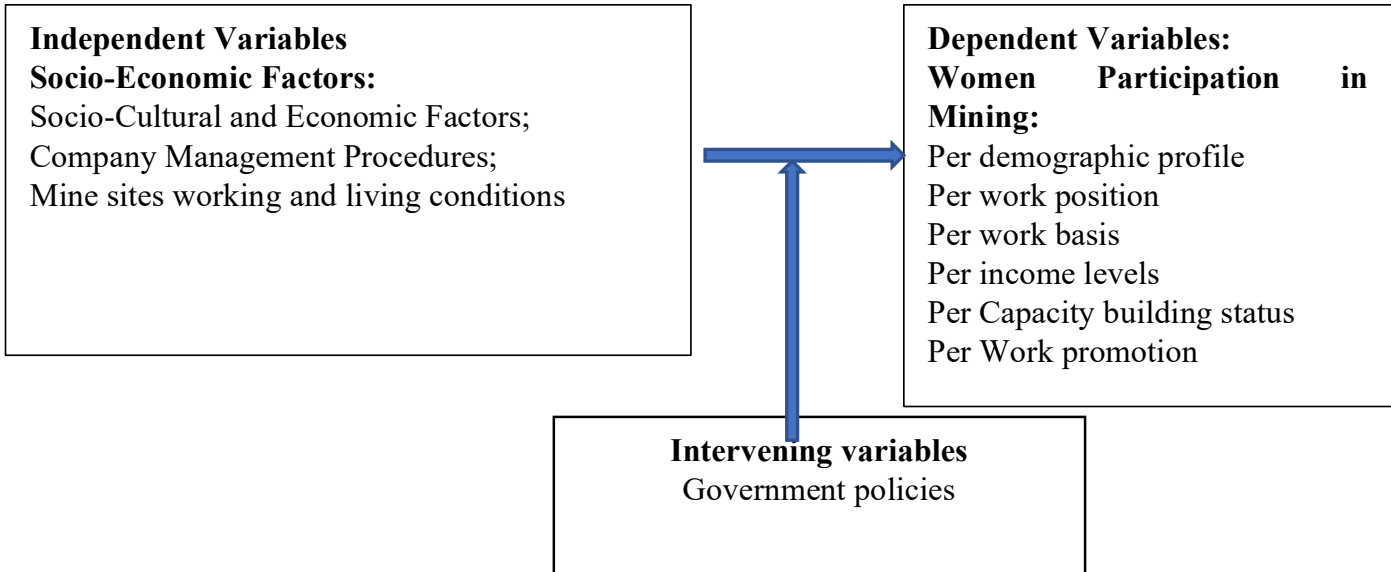

Figure1: Conceptual framework

\section{6. $\quad$ Research design}

This research has used descriptive and correlational research designs. According to (Jayanka K.N and Priyanka S., 2015),

\subsection{Target population}

The target population was comprised by the total population of 835 including 676 men and 159 women miners.

\subsection{Sample Size}

Slovene's formula was used to determine the sample size (Fadilah P. and Mohd H., 2017) with the error tolerance of $5 \%$. Using the formula:

$$
n=\frac{N}{1+N e^{2}}
$$


Where: $\mathrm{n}=$ Number of samples; $\mathrm{N}=$ Total population and $\mathrm{e}=$ the margin of error estimated at $5 \%$.

$n=\frac{835}{1+835 * 0.05 * 0.05}=270$

To determine the number of men or women included in the sample from each selected company, proportion to size method has been used.

Table1: Sample population

\begin{tabular}{|c|c|c|c|c|c|c|c|c|c|c|}
\hline \multirow[t]{2}{*}{ Province } & \multirow[t]{2}{*}{$\begin{array}{l}\text { Compa } \\
\text { nies }\end{array}$} & \multicolumn{3}{|c|}{ Target population } & \multicolumn{3}{|c|}{ Percentage } & \multicolumn{3}{|c|}{ Sample } \\
\hline & & Total & $\begin{array}{l}\text { Mal } \\
\text { e }\end{array}$ & $\begin{array}{l}\text { Femal } \\
\text { e }\end{array}$ & Total & Male & Female & Total & Male & Female \\
\hline \multirow{4}{*}{ Kigali } & 1 & 28 & 24 & 4 & 3 & 86 & 14 & 9 & 8 & 1 \\
\hline & 2 & 25 & 20 & 5 & 3 & 80 & 20 & 8 & 6 & 2 \\
\hline & 3 & 21 & 17 & 4 & 3 & 81 & 19 & 7 & 6 & 1 \\
\hline & 4 & 33 & 28 & 5 & 4 & 85 & 15 & 11 & 9 & 2 \\
\hline \multirow[t]{8}{*}{ South } & 1 & 32 & 27 & 5 & 4 & 84 & 16 & 10 & 9 & 1 \\
\hline & 2 & 30 & 26 & 4 & 4 & 87 & 13 & 10 & 8 & 2 \\
\hline & 3 & 36 & 28 & 8 & 4 & 78 & 22 & 12 & 9 & 3 \\
\hline & 4 & 35 & 28 & 7 & 4 & 80 & 20 & 11 & 9 & 2 \\
\hline & 5 & 36 & 32 & 4 & 4 & 89 & 11 & 12 & 10 & 2 \\
\hline & 6 & 24 & 20 & 4 & 3 & 83 & 17 & 8 & 6 & 2 \\
\hline & 7 & 27 & 23 & 4 & 3 & 85 & 15 & 9 & 7 & 2 \\
\hline & 8 & 22 & 19 & 3 & 3 & 86 & 14 & 7 & 6 & 1 \\
\hline \multirow[t]{6}{*}{ West } & 1 & 32 & 26 & 6 & 4 & 81 & 19 & 10 & 8 & 2 \\
\hline & 2 & 18 & 14 & 4 & 2 & 78 & 22 & 6 & 5 & 1 \\
\hline & 3 & 24 & 20 & 4 & 3 & 83 & 17 & 8 & 6 & 2 \\
\hline & 4 & 19 & 15 & 4 & 2 & 79 & 21 & 6 & 5 & 1 \\
\hline & 5 & 21 & 18 & 3 & 3 & 86 & 14 & 7 & 6 & 1 \\
\hline & 6 & 22 & 17 & 5 & 3 & 77 & 23 & 7 & 6 & 1 \\
\hline \multirow[t]{5}{*}{ North } & 1 & 25 & 20 & 5 & 3 & 80 & 20 & 8 & 6 & 2 \\
\hline & 2 & 23 & 17 & 6 & 3 & 74 & 26 & 7 & 6 & 1 \\
\hline & 3 & 24 & 15 & 9 & 3 & 63 & 37 & 8 & 5 & 3 \\
\hline & 4 & 28 & 23 & 5 & 3 & 82 & 18 & 9 & 7 & 2 \\
\hline & 5 & 36 & 30 & 6 & 4 & 83 & 17 & 12 & 10 & 2 \\
\hline \multirow[t]{7}{*}{ East } & 1 & 24 & 20 & 4 & 3 & 83 & 17 & 8 & 7 & 1 \\
\hline & 2 & 23 & 20 & 3 & 3 & 87 & 13 & 7 & 6 & 1 \\
\hline & 3 & 34 & 27 & 7 & 4 & 79 & 21 & 11 & 9 & 2 \\
\hline & 4 & 24 & 20 & 4 & 3 & 83 & 17 & 7 & 6 & 1 \\
\hline & 5 & 32 & 23 & 9 & 4 & 72 & 28 & 10 & 9 & 1 \\
\hline & 6 & 41 & 31 & 10 & 5 & 76 & 24 & 13 & 10 & 3 \\
\hline & 7 & 36 & 28 & 8 & 4 & 78 & 22 & 12 & 9 & 3 \\
\hline Total & 30 & 835 & 676 & 159 & 100 & 81 & 19 & 270 & 219 & 51 \\
\hline
\end{tabular}

Source: (RMB, 2019) and Researcher's calculations 


\subsection{Sampling Techniques}

Purposive and simple random sampling techniques were used to select respondents.

\section{Data collection Methods}

Primary and secondary data sources were used to gather information in this research.

\subsection{Data collection Instruments}

Questionnaires were used as instruments for data collection.

\section{Research Findings and Discussions}

\subsection{Presentation of Findings}

Findings from the research are organized, analyzed and presented per objective. Each objective has its specific data collected to answer associated questions.

\subsubsection{Investigating the effect of Socio-cultural and economic factors on women participation in mining}

This research objective was achieved by using descriptive analysis for statistical data collected from respondents' perceptions on socio-cultural and economic factors as shown in the table 2 and 3 respectively.

Table2: Perceptions on Socio-cultural factors and women participation in mining

\begin{tabular}{|c|c|c|c|c|c|c|}
\hline \multirow[b]{2}{*}{ Perceptions } & \multicolumn{2}{|c|}{ Female } & \multicolumn{2}{|c|}{ Male } & \multicolumn{2}{|c|}{ Total } \\
\hline & $\begin{array}{c}\text { Frequenc } \\
\mathbf{y}\end{array}$ & $\begin{array}{l}\text { Percent } \\
\text { age }\end{array}$ & $\begin{array}{c}\text { Frequen } \\
\text { cy }\end{array}$ & $\begin{array}{l}\text { Percent } \\
\text { age }\end{array}$ & $\begin{array}{l}\text { Frequen } \\
\text { cy }\end{array}$ & $\begin{array}{l}\text { Percent } \\
\text { age }\end{array}$ \\
\hline \multicolumn{7}{|c|}{ 1. Could you work in mining if you had alternative job? } \\
\hline No & 48 & 94.1 & 6 & 2.7 & 54 & 20 \\
\hline Yes & 3 & 5.9 & 213 & 97.3 & 216 & 80 \\
\hline Total & 51 & 100.0 & 219 & 100.0 & 270 & 100.0 \\
\hline \multicolumn{7}{|l|}{ 2. If No why? } \\
\hline $\begin{array}{l}\text {-Because combining household } \\
\text { responsibilities with mining is very hard }\end{array}$ & 9 & 19.1 & 0 & 0 & 9 & 17.0 \\
\hline $\begin{array}{l}\text {-Because Mining is very hard and risky for } \\
\text { fatal accidents }\end{array}$ & 6 & 12.8 & 6 & 100 & 12 & 22.6 \\
\hline $\begin{array}{l}\text {-Because, community perceived me as a } \\
\text { prostitute }\end{array}$ & 12 & 25.5 & 0 & 0 & 12 & 22.6 \\
\hline $\begin{array}{l}\text {-Because my work in mining was seen as } \\
\text { against culture }\end{array}$ & 20 & 42.6 & 0 & 0 & 20 & 37.7 \\
\hline Total & 47 & 100.0 & 6 & 100 & 53 & 100.0 \\
\hline \multicolumn{7}{|c|}{ 3. If you earn much income, what will you do in future? } \\
\hline -Continue working in mining & 6 & 11.8 & 203 & 92.7 & 209 & 77.4 \\
\hline -Leave mining for other businesses & 45 & 88.2 & 16 & 7.3 & 61 & 22.6 \\
\hline Total & 51 & 100.0 & 219 & 100.0 & 270 & 100.0 \\
\hline
\end{tabular}

Source: Field data, 2019

The table 2 indicates that a big majority of women (94.1\%) join mining activity not because they like it but because of poverty with no other alternative means to survive. Employment of women in mining is challenged with sociocultural beliefs where $42.6 \%$ of females in this research confirmed that employment of women in mining is seen as against culture, while others $(25.5 . \%)$ confirmed that community perceives them as prostitute. 
Table3. Income distribution between men and women per mining work basis

\begin{tabular}{|c|c|c|c|c|c|c|}
\hline \multirow[b]{2}{*}{ Perceptions } & \multicolumn{2}{|c|}{ Female } & \multicolumn{2}{|c|}{ Male } & \multicolumn{2}{|c|}{ Total } \\
\hline & Freq. & Perc. & Freq. & Perc. & Freq. & Perc. \\
\hline \multicolumn{7}{|l|}{ 1. What is your work basis? } \\
\hline Casual basis & 46 & 90.2 & 56 & 25.6 & 102 & 37.8 \\
\hline Permanent basis/Monthly & 5 & 9.8 & 163 & 74.4 & 168 & 62.2 \\
\hline Total & 51 & 100.0 & 219 & 100.0 & 270 & 100.0 \\
\hline \multicolumn{7}{|c|}{ 2. If casual how much do you earn per day? } \\
\hline 1000 & 36 & 78.3 & 0 & 0 & 33 & 33.3 \\
\hline 1500 & 9 & 19.6 & 3 & 5.4 & 12 & 12.1 \\
\hline 2000 & 1 & 2.2 & 8 & 14.3 & 9 & 9.1 \\
\hline 2500 & 0 & 0 & 4 & 7.1 & 4 & 4.0 \\
\hline 3000 & 0 & 0 & 15 & 26.8 & 15 & 15.2 \\
\hline 3500 & 0 & 0 & 8 & 14.3 & 8 & 8.1 \\
\hline 4000 & 0 & 0 & 7 & 12.5 & 7 & 7.1 \\
\hline 4500 & 0 & 0 & 6 & 10.7 & 6 & 6.1 \\
\hline 5000 & 0 & 0 & 5 & 8.9 & 5 & 5.1 \\
\hline Total & 46 & 100.0 & 56 & 100.0 & 99 & 100.0 \\
\hline \multicolumn{7}{|c|}{ 3. If permanent, how much do you earn per month? } \\
\hline 25,000 & 3 & 60 & 0 & 0 & 3 & 1.8 \\
\hline 30,000 & 1 & 20 & 6 & 3.7 & 7 & 4.2 \\
\hline 35,000 & 0 & 0 & 8 & 4.9 & 8 & 4.8 \\
\hline 40,000 & 0 & 0 & 5 & 3.1 & 5 & 3.0 \\
\hline 45,000 & 0 & 0 & 11 & 6.7 & 11 & 6.5 \\
\hline 50,000 & 0 & 0 & 15 & 9.2 & 15 & 8.9 \\
\hline 55,000 & 0 & 0 & 12 & 7.4 & 12 & 7.1 \\
\hline 60,000 & 1 & 20 & 21 & 12.9 & 22 & 13.1 \\
\hline 65,000 & 0 & 0 & 0 & 0 & 0 & 0.0 \\
\hline 70,000 & 0 & 0 & 19 & 11.7 & 19 & 11.3 \\
\hline 75,000 & 0 & 0 & 18 & 11.0 & 18 & 10.7 \\
\hline 80,000 & 0 & 0 & 10 & 6.1 & 10 & 6.0 \\
\hline 85,000 & 0 & 0 & 22 & 13.5 & 22 & 13.1 \\
\hline 90,000 & 0 & 0 & 5 & 3.1 & 5 & 3.0 \\
\hline 95,000 & 0 & 0 & 7 & 4.3 & 7 & 4.2 \\
\hline 100,000 & 0 & 0 & 4 & 2.5 & 4 & 2.4 \\
\hline Total & 5 & 100 & 163 & 100 & 168 & 100.0 \\
\hline \multicolumn{7}{|c|}{ 3. At which age would you leave mining? } \\
\hline 40 & 43 & 84.3 & 0 & 0 & 43 & 15.9 \\
\hline 45 & 7 & 13.7 & 15 & 6.8 & 22 & 8.1 \\
\hline 50 & 1 & 2.0 & 93 & 42.5 & 94 & 34.8 \\
\hline 55 & 0 & 0.0 & 100 & 45.7 & 100 & 37.0 \\
\hline 60 & 0 & 0.0 & 11 & 5.0 & 11 & 4.1 \\
\hline Total & 51 & 100.0 & 219 & 100 & 270 & 100.0 \\
\hline
\end{tabular}

Source: Field data, 2019

The table 3 indicates a big difference between males and females' earnings from mining. Majority of male workers (74.4\%) work on permanent basis which majority of them (13.5\%) get a monthly salary of 85,000 Rwf. Women who work on permanent basis represent $9.8 \%$ only but however their monthly salary is very low comparing to that of permanent men workers as majority of permanent women $(60 \%)$ get a monthly salary of 25,000 Frw only. 
Table 4: Chi-square and regression results for objective 1

\begin{tabular}{|c|c|c|c|c|c|c|c|}
\hline \multirow[b]{2}{*}{ Predictors } & \multicolumn{2}{|c|}{ No } & \multicolumn{2}{|c|}{ Yes } & \multicolumn{2}{|c|}{ Total } & \multirow[b]{2}{*}{ P value } \\
\hline & $\begin{array}{l}\text { Frequen } \\
\text { cy }\end{array}$ & $\begin{array}{l}\text { Percentag } \\
\text { e }\end{array}$ & $\begin{array}{l}\text { Frequenc } \\
\text { y }\end{array}$ & $\begin{array}{l}\text { Percentag } \\
\text { e }\end{array}$ & $\begin{array}{l}\text { Frequenc } \\
\text { y }\end{array}$ & $\begin{array}{l}\text { Percent } \\
\text { age }\end{array}$ & \\
\hline \multicolumn{8}{|c|}{ 1. Do you think that social responsibilities affect your participation in mining } \\
\hline Female & 7 & 13.7 & 44 & 86.3 & 51 & 100 & \multirow[b]{3}{*}{0.000} \\
\hline Male & 204 & 93.2 & 15 & 6.8 & 219 & 100 & \\
\hline Total & 211 & 78.1 & 59 & 21.9 & 270 & 100 & \\
\hline \multicolumn{8}{|c|}{ 2. Do cultural beliefs in Rwanda support your employment in mining? } \\
\hline Female & 50 & 98 & 1 & 2 & 51 & 100 & \multirow[b]{3}{*}{0.000} \\
\hline Male & 10 & 4.6 & 209 & 95.4 & 219 & 100 & \\
\hline Total & 60 & 22.2 & 210 & 77.8 & 270 & 100 & \\
\hline \multicolumn{7}{|c|}{ 3. Could you work in mining if you had other sources of income? } & \\
\hline Female & 47 & 92.2 & 4 & 7.8 & 51 & 100 & \multirow[b]{3}{*}{0.000} \\
\hline Male & 5 & 2.3 & 214 & 97.7 & 219 & 100 & \\
\hline Total & 52 & 19.3 & 218 & 80.7 & 270 & 100 & \\
\hline
\end{tabular}

Regression coefficients for the objective 1

\begin{tabular}{|l|r|r|r|r|r|r|r|}
\hline Predictors & \multicolumn{1}{c}{ Coef. } & \multicolumn{1}{c}{ Std. Err. } & \multicolumn{1}{c}{ P $>t$} & \multicolumn{1}{c}{ [95\% Conf. } & Interval] \\
\hline Social responsibilities & 0.049 & 0.0335 & 1.46 & 0.145 & -0.017 & 0.1149 \\
\hline Cultural beliefs & -0.36 & 0.0382 & -9.45 & 0 & -0.436 & -0.285 \\
\hline Sources of income & -0.433 & 0.0433 & -10.01 & 0 & -0.518 & -0.348 \\
\hline cons & 0.7811 & 0.0392 & 19.93 & 0 & 0.704 & 0.8582 \\
\hline
\end{tabular}

Source: researcher's calculations, 2020

The regression model in form of $Y=\alpha_{0}+\alpha_{1} x_{1}+\alpha_{2} x_{2}+\alpha_{3} x_{3}+\varepsilon$ becomes:

$\mathrm{WP}=0.78+0.049$ SR $-0.36 \mathrm{CB}-0.43 \mathrm{SI}+\varepsilon$; Where WP $=$ Women Participation $(Y)$; SR: Social Responsibilities $\left(X_{1}\right)$ with a coefficient of. 0.049; CB: Cultural Beliefs $\left(X_{2}\right)$ with (coef. $\left.=-0.36\right)$ and SI: Sources of Income $\left(X_{3}\right)$ with a coefficient of -0.43 and the constant of 0.78 . The chi- square results show that predictor variable related to social responsibilities for the objective one, has a positive effect(coef. 0.049 ) on women participation while cultural beliefs (coef -0.36) and sources of income (-0.43) have a negative relationship with women participation in mining. As social responsibilities in terms of fulfilling household needs increase with no alternative jobs in rural areas, more women especially single mothers tend to join mining as the only off-farm employment. On the other hand, as much as there is an increase of people who believe in cultural norms and traditions where employment of women in mining is negatively perceived by community when the number of alternative sources of income increase, the number of women who join mining will decrease and vice versa.

\subsubsection{Determining the influence of company management procedures on women participation in mining projects.}

Assessing the effect of company management procedures on women participation in mining started by compiling data from different reports in mining companies' administration mainly focusing on domains involved in the management of workers as well as from respondents' perceptions about human resources management procedures vis a vis gender inclusion. 
Table5: Respondents perceptions on Human resources Management

\begin{tabular}{|c|c|c|c|c|c|c|}
\hline \multirow[t]{2}{*}{ Perceptions } & \multicolumn{3}{|c|}{ Frequency } & \multicolumn{3}{|c|}{ Percentage } \\
\hline & Male & Female & Total & Male & Female & Total \\
\hline \multicolumn{7}{|c|}{ 1. What are the main challenges have you ever got during recruitment in mining? } \\
\hline $\begin{array}{l}\text { My candidature was not easily accepted by } \\
\text { company management }\end{array}$ & 0 & 6 & 6 & 0.0 & 11.8 & 2.2 \\
\hline $\begin{array}{l}\text { My abilities to work in some mining } \\
\text { activities was undermined by management }\end{array}$ & 3 & 14 & 17 & 1.4 & 27.5 & 6.3 \\
\hline $\begin{array}{l}\text { Required conditions to be recruited were } \\
\text { complicated }\end{array}$ & 0 & 22 & 22 & 0.0 & 43.1 & 8.1 \\
\hline $\begin{array}{l}\text { Lack of information about vacant jobs in } \\
\text { mining }\end{array}$ & 56 & 7 & 63 & 25.6 & 13.7 & 23.3 \\
\hline No challenge & 160 & 2 & 162 & 73.1 & 3.9 & 60.0 \\
\hline Total & 219 & 51 & 270 & 100 & 100.0 & 100.0 \\
\hline \multicolumn{7}{|l|}{ 2. In which activity are you allocated? } \\
\hline Mineral washing and panning & 14 & 54 & 68 & 27.5 & 24.7 & 25.2 \\
\hline Carrier of water/ore materials & 31 & 75 & 106 & 60.8 & 34.2 & 39.3 \\
\hline Digging & 0 & 40 & 40 & - & 18.3 & 14.8 \\
\hline Ground sluicing & 0 & 24 & 24 & - & 11.0 & 8.9 \\
\hline Mine technicians & 2 & 2 & 4 & 3.9 & 0.9 & 1.5 \\
\hline Ore grinding & 2 & 14 & 16 & 3.9 & 6.4 & 5.9 \\
\hline Support services & 2 & 10 & 12 & 3.9 & 4.6 & 4.4 \\
\hline Total & 51 & 219 & 270 & 100.0 & 100.0 & 100.0 \\
\hline \multicolumn{7}{|c|}{ 2. What would be the most reason for you to be allocated in low paid jobs? } \\
\hline $\begin{array}{l}\text { Managers undermine my capabilities to } \\
\text { perform high paid jobs }\end{array}$ & 11 & 19 & 30 & 5.0 & 37.3 & 11.1 \\
\hline $\begin{array}{l}\text { High paid jobs require physical strength, } \\
\text { skills and knowledge that I don't have }\end{array}$ & 53 & 18 & 71 & 24.2 & 35.3 & 26.3 \\
\hline $\begin{array}{l}\text { I don't feel confident to apply for high paid } \\
\text { works }\end{array}$ & 5 & 14 & 19 & 2.3 & 27.5 & 7.0 \\
\hline I am not concerned with low paid jobs & 150 & 0 & 150 & 68.5 & 0.0 & 55.6 \\
\hline Total & 219 & 51 & 270 & 100.0 & 100.0 & 100.0 \\
\hline \multicolumn{7}{|l|}{ 3. In which activity have you ever been trained? } \\
\hline Blasting & 14 & 0 & 14 & 6.4 & 0.0 & 5.2 \\
\hline Digging & 57 & 0 & 57 & 26.0 & 0.0 & 21.1 \\
\hline Ground sluicing & 82 & 0 & 82 & 37.4 & 0.0 & 30.4 \\
\hline Grinding & 41 & 2 & 43 & 18.7 & 3.9 & 15.9 \\
\hline Panning & 22 & 8 & 30 & 10.0 & 15.7 & 11.1 \\
\hline No Training obtained & 3 & 41 & 44 & 1.4 & 80.4 & 16.3 \\
\hline Total & 219 & 51 & 270 & 100.0 & 100.0 & 100.0 \\
\hline \multicolumn{7}{|c|}{ 4. Have you ever been promoted from low to high paid job? } \\
\hline No & 40 & 49 & 89 & 18.3 & 96.1 & 33.0 \\
\hline Yes & 179 & 2 & 181 & 81.7 & 3.9 & 67.0 \\
\hline Total & 219 & 51 & 270 & 100.0 & 100.0 & 100.0 \\
\hline
\end{tabular}

Source: field data, 2019

The table 5 shows that different procedures applied in human resources management such as recruitment procedure, job allocation system, promotion procedures, skills development procedures, etc., are not favorable for the majority of women miners. Respondents confirmed that conditions set out for women to be recruited in mining jobs are 
complicated and even though they are recruited, they are allocated in low paid jobs because they don't have required knowledge and skills for high paid jobs. Besides, women in mining do get the same chance for training as their men co-workers. The other issue is that even though women get the same knowledge and experience as men, company management undermines their capabilities to perform some mining activities as men. Promotion rate is very low for women miners (3.9\%) comparing to that of men co-workers $(81.7 \%)$

Using chi-square test and regression models, through SPSS software, independent variables have been tested to check whether they are correlated with dependent variable or not.

Table6: Chi-square and regression results for objective 2

\begin{tabular}{|c|c|c|c|c|c|c|c|}
\hline \multirow[b]{2}{*}{ Predictors } & \multicolumn{2}{|c|}{ No } & \multicolumn{2}{|c|}{ Yes } & \multicolumn{2}{|c|}{ Total } & \multirow[b]{2}{*}{ P-value } \\
\hline & $\begin{array}{l}\text { Freque } \\
\text { ncy }\end{array}$ & $\begin{array}{l}\text { Percentag } \\
\text { e }\end{array}$ & $\begin{array}{l}\text { Freque } \\
\text { ncy }\end{array}$ & $\begin{array}{l}\text { Percen } \\
\text { tage }\end{array}$ & $\begin{array}{l}\text { Freque } \\
\text { ncy }\end{array}$ & $\begin{array}{l}\text { Percen } \\
\text { tage }\end{array}$ & \\
\hline \multicolumn{8}{|c|}{$\begin{array}{l}\text { 1. Do you think recruitment procedures are favorable for your } \\
\text { participation in mining }\end{array}$} \\
\hline Female & 47 & 92.2 & 4 & 7.8 & 51 & 100 & \multirow[b]{3}{*}{0.000} \\
\hline Male & 14 & 6.5 & 203 & 93.5 & 217 & 100 & \\
\hline Total & 61 & 22.8 & 207 & 77.2 & 268 & 100 & \\
\hline \multicolumn{8}{|c|}{ 2. Do you think job allocation procedures are favorable for your participation in mining? } \\
\hline Female & 40 & 78.4 & 11 & 21.6 & 51 & 100 & \multirow[b]{3}{*}{0.000} \\
\hline Male & 15 & 7 & 200 & 93 & 215 & 100 & \\
\hline Total & 55 & 20.7 & 211 & 79.3 & 266 & 100 & \\
\hline \multicolumn{7}{|c|}{$\begin{array}{l}\text { 3. Do you think skills development procedures support your employment in } \\
\text { mining? }\end{array}$} & \\
\hline Female & 49 & 96.1 & 2 & 3.9 & 51 & 100 & \multirow[b]{3}{*}{0.000} \\
\hline Male & 0 & 0 & 216 & 100 & 216 & 100 & \\
\hline Total & 49 & 18.4 & 218 & 81.6 & 267 & 100 & \\
\hline \multicolumn{8}{|c|}{ 4. Do you think Employee promotion procedures are favorable your participation in mining? } \\
\hline Female & 39 & 79.6 & 10 & 20.4 & 49 & 100 & \multirow[b]{3}{*}{0.000} \\
\hline Male & 52 & 23.7 & 167 & 76.3 & 219 & 100 & \\
\hline Total & 91 & 34 & 177 & 66 & 268 & 100 & \\
\hline
\end{tabular}

Regression coefficient for the objective 2

\begin{tabular}{|c|c|c|c|c|c|c|}
\hline Predictors & Coef. & $\begin{array}{l}\text { Std. } \\
\text { Err. }\end{array}$ & $\mathbf{t}$ & $\mathbf{P}>\mathbf{t}$ & $\begin{array}{l}{[95 \%} \\
\text { Conf. }\end{array}$ & $\begin{array}{l}\text { Interva } \\
\text { l] }\end{array}$ \\
\hline Recruitment procedures (RP) & -0.0483 & 0.0212 & -2.28 & 0.024 & -0.09 & -0.006 \\
\hline Job allocation procedures (JAP) & -0.0343 & 0.0182 & -1.88 & 0.061 & -0.07 & 0.0016 \\
\hline Skills Development Procedures (SDP) & -0.919 & 0.0266 & -34.49 & 0 & -0.97 & -0.866 \\
\hline Employee Promotion Procedures (EPP) & -0.0095 & 0.0128 & -0.74 & 0.458 & -0.03 & 0.0157 \\
\hline _constant & 1.0122 & 0.0135 & 75.24 & 0 & 0.986 & 1.0387 \\
\hline
\end{tabular}

Source: Researchers calculations, 2020

Using the regression model, we have:

$\mathrm{WP}=1.01-0.0483$ SDP $-0.0343 \mathrm{WDP}-0.919 \mathrm{EPP}-0.009+\varepsilon$

(WP: Women Participation; SDP:Skills Development Procedures; EPP: Employee Promotion Procedures).

The model shows that comparing to other independent variables, skills development procedures with the coefficient of -0.919 present high negative relationships with the dependent variable. This implies that if no improvement is done on current skills development procedures, women participation will decrease more and more. 


\subsubsection{Examining the impact of mine site working and living conditions and women participation in mining.}

This objective was assessed based on respondents' perceptions about the status of mining work places as well as their working and living conditions on mine sites.

The table 7: Challenges of mining nature and working and living conditions.

\begin{tabular}{|c|c|c|c|c|c|c|}
\hline \multirow[b]{2}{*}{ Perceptions } & \multicolumn{2}{|c|}{ Female } & \multicolumn{2}{|c|}{ Male } & \multicolumn{2}{|c|}{ Total } \\
\hline & $\begin{array}{c}\text { Freque } \\
\text { ncy }\end{array}$ & $\begin{array}{l}\text { Percenta } \\
\text { ge }\end{array}$ & $\begin{array}{c}\text { Freque } \\
\text { ncy }\end{array}$ & $\begin{array}{c}\text { Percen } \\
\text { tage }\end{array}$ & $\begin{array}{c}\text { Frequenc } \\
\mathbf{y}\end{array}$ & $\begin{array}{c}\text { Percenta } \\
\text { ge }\end{array}$ \\
\hline \multicolumn{7}{|c|}{ 1. Do you think that mining work places present particular challenges for your work? } \\
\hline No & 3 & 5.9 & 211 & 96.3 & 214 & 79.3 \\
\hline Yes & 48 & 94.1 & 8 & 3.7 & 56 & 20.7 \\
\hline Total & 51 & 100.0 & 219 & 100.0 & 270 & 100.0 \\
\hline \multicolumn{7}{|l|}{ 2. If yes what are they? } \\
\hline Fear for tentative sexual violence & 10 & 20.8 & 0 & 0 & 10 & 17.9 \\
\hline Fatal accidents & 15 & 31.3 & 6 & 75 & 21 & 37.5 \\
\hline Fear for walking long distance & 23 & 47.9 & 2 & 25 & 25 & 44.6 \\
\hline Total & 48 & 100.0 & 8 & 100 & 56 & 100.0 \\
\hline \multicolumn{7}{|c|}{ 3. Are many working hours a challenge for your work in mining? } \\
\hline No & 8 & 16 & 213 & 97.3 & 221 & 81.9 \\
\hline Yes & 43 & 84 & 6 & 2.7 & 49 & 18.148 \\
\hline Total & 51 & 100 & 219 & 100.0 & 270 & 100.0 \\
\hline \multicolumn{7}{|c|}{ 4. Do you think that OHS basic rights associated with your work are respected? } \\
\hline No & 45 & 88.24 & 15 & 6.8 & 60 & 22.2 \\
\hline Yes & 6 & 11.76 & 204 & 93.2 & 210 & 77.8 \\
\hline Total & 51 & 100.00 & 219 & 100.0 & 270 & 100.0 \\
\hline \multicolumn{7}{|c|}{ 5. If no which of your rights are violated? } \\
\hline Being fired when you are pregnant & 12 & 26.7 & 0 & 0 & 12 & 21.1 \\
\hline Lack of contract & 30 & 66.7 & 15 & 100 & 45 & 78.9 \\
\hline Lack of necessary facilities & 3 & 6.7 & 0 & 0 & 0 & 0.0 \\
\hline Total & 45 & 100.0 & 15 & 100 & 57 & 100.0 \\
\hline \multicolumn{7}{|l|}{ 6. Do you have work contracts? } \\
\hline No & 47 & 92.2 & 12 & 5.5 & 59 & 21.8 \\
\hline Yes & 4 & 7.8 & 207 & 94.5 & 211 & 78.2 \\
\hline Total & 51 & 100 & 219 & 100 & 270 & 100 \\
\hline
\end{tabular}

Source: field data, 2019

The table 7 indicates that of $94.1 \%$ of female workers confirm that mining work places present particular challenges for their work mostly due to the nature of mine site's location. In fact, majority of women (47.9\%) confirmed that they fear to walk long distance from work place to their home while others $(31.3 \%)$ fear working in underground shafts. The issue of tentative sexual harassment when working in underground has been also said to be a challenge for $20.8 \%$ of female respondents. On the side of males, majority of them $(96.3 \%)$ confirmed that mining activity does not present any challenge for them.

Regarding the Occupational Safety and Health conditions (OSH), the table 7 shows some work basic rights are not respected for the majority $(88.24 \%)$ in such way that $92.3 \%$ of women respondents do not have work contracts while $26.7 \%$ confirmed that being fired once become pregnant is a challenge for them.

Using scientific method through chi-square and multiple regression analysis, two predictors, the nature of mining activity and the OSH conditions applied in mining were assessed to check, correlation and effect between independent and dependent variables. 
Table8: Chi-square and regression results, Objective 3

\begin{tabular}{|c|c|c|c|c|c|c|c|}
\hline \multirow[b]{2}{*}{ Sex } & \multicolumn{2}{|r|}{ No } & \multicolumn{2}{|c|}{ Yes } & \multicolumn{2}{|c|}{ Total } & \multirow[b]{2}{*}{ P value } \\
\hline & $\begin{array}{l}\text { Frequen } \\
\text { cy }\end{array}$ & Percentage & $\begin{array}{l}\text { Frequenc } \\
\mathrm{y}\end{array}$ & $\begin{array}{l}\text { Percentag } \\
\text { e }\end{array}$ & $\begin{array}{l}\text { Frequenc } \\
\mathrm{y}\end{array}$ & Percentage & \\
\hline \multicolumn{8}{|c|}{ 1. Is the nature of mining work places favorable for improving your livelihood? } \\
\hline Male & 75 & & 144 & & 219 & 100 & \multirow[b]{3}{*}{0.000} \\
\hline Female & 38 & & 13 & & 51 & 100 & \\
\hline Total & 113 & & 157 & & 270 & 100 & \\
\hline \multicolumn{8}{|c|}{ 2. OSH conditions are favorable for your participation in mining? } \\
\hline Male & 77 & & 142 & & 219 & 100 & \multirow[b]{3}{*}{0.000} \\
\hline Female & 41 & & 10 & & 51 & 100 & \\
\hline Total & 118 & & 152 & & 270 & 100 & \\
\hline
\end{tabular}

Regression coefficients for the objective 3

\begin{tabular}{|l|l|}
\hline Predictors & Coefficients. \\
\hline Nature of Mining workplace (NMW) & $-0.154625(\approx-0.15)$ \\
\hline OSH conditions (OSH) & $-0.195156(\approx-0.19)$ \\
\hline Constant & $0.156156(\approx 0.16)$ \\
\hline
\end{tabular}

Source: Researcher's calculations

The multiple regression model in the form of: $Y=\delta_{0}+\delta_{1} t_{1}+\delta_{2} t_{2}+\varepsilon$ for the objective 3 becomes WP $=\varepsilon$ $0.15 \mathrm{NMW}-0.19 \mathrm{OSH}+0.16$

Where $\mathrm{WP}=$ Women Participation; NMW $=$ Nature of mining workplaces and $\mathrm{OSH}=$ Occupational Safety and Health.

Both two predictors affect women participation negatively and it is shown that the OSH predictor has a high negative effect on women participation comparing to the nature of mining work. This would mean that at the extent working and living conditions become more deplorable, the participation of women in mining decreases and vice versa.

\section{Conclusion and Recommendations}

\subsection{Conclusion}

Since 1930 when mining started till now, Socio-cultural factors have been forcing minds of Rwandan community to behave in a way to conclude that mining is for men not for women and hence consider gender inequality in mining as inevitable. Procedures applied in human resources management of mining companies do not at all leave audience to women for joining mining. Socio-cultural factors in terms of social responsibilities and cultural beliefs as well as demographic factors in terms of marital status (married, widowed, single, single mothers, etc.,), affect women participation in mining either positively or negatively. Nature of mining work-places and OSH conditions not favourable for women do not allow them to feel mining as easier for them and prefer not joining it.

If mining is to contribute for poverty alleviation in rural areas and especially eliminating inequality between men and women in accessing economic benefits in rural areas, all these factors which affect women participation in mining negatively should be addressed in a favourable manner to women.

\subsection{Recommendations}

Mobilization at community level in general and at company level in particular aiming at improving common understanding about equal sharing of economic resources between men and women would help community to change their mind on equal role on performing economic activities.

For effective social inclusion in benefiting from mining resources, all components of human resources 
management should reflect gender equality, starting from workers recruitment planning, allocation of jobs to workers, skills development, workers promotion and workers retention strategies should focus to both men and women.

Strong strategies should be put in place by mining regulators to address poor OSH conditions especially associated with employment of women in mining in order to ensure that gender principles are considered as a standard for mining best practice.

Study trips and peer learning methods between mining companies with good records about gender inclusion in their mining operations and those with poor records can be used.

Improved working and living conditions of mine work places in terms of establishing positive work environment for all workers and especially for women is an important input to integrate more women in Rwandan mining projects.

\section{References}

Fadilah P. and Mohd H. (2017). Quantitative analysis: Choosing between SPSS, PLS and AMOS in social science research. Mara-Malaysia: International Interdisciplinary Journal of Scientific Research .

ICGLR. (2017). Assessment Study on Gender Mainstreaming in the Mining Sector, opportunities and challenges in promoting Gender Equality with focus on Artisanal Small Scale Mining in The Central Africa Republic and the Republic of Rwanda. Bujumbura: ICGLR Secretariat.

Jayanka K.N and Prianka S. (2015). Fandamentals of Research Methodology, Problems and Prospects. New Delhi: SSDN Pubrishers \& Distributors.

MIHRC. (2016). Strengthening Mining's Talent Alloy: Exploring Gender inclusion. Kanata, Ontario: MIHRC.

MINECOFIN. (2013). Economic development and poverty reduction strategy. Kigali: MINECOFIN.

NISR. (2014). Fourth population and housing census 2012, Census Atlas. Kigali: NISR.

Oxfam International. (2017). Position Paper on gender Justice and the Extractive Industry. Washington D.C: Oxfam America.

Pimpa, N. (2019). How mining companies promote gender equality through sustainable development? Cogent Business \& Management, 01-06.

RMB. (2019). Mining data base 2018. Kigali: RMB.

RNRA. (2016). Mining Statistics. Kigali: RNRA. 